\title{
EL FACTOR DIFERENCIAL DE LA MIGRACIÓN EN LAS PERSONAS SIN HOGAR
}

\section{THE DISTINGUISHING FACTOR OF MIGRATION IN HOMELESSNESS}

Miguel Ángel Navarro-Lashayas*

Resumen: En los últimos años ha crecido el número de personas inmigrantes sin hogar. El artículo muestra las relaciones entre la situación documental y el estrés aculturativo con variables sociodemográficas, apoyo social, abusos de sustancias, sucesos vitales estresantes y malestar emocional en una muestra de 107 personas migrantes sin hogar. Para ello se han realizado entrevistas en profundidad y autoinformes analizando las relaciones entre las variables mediante diversas técnicas de análisis de datos y análisis de contenido. Los resultados manifiestan la importancia de lo cualitativo para la variable «situación documental» que apenas tiene correlaciones con el resto de las variables. También destaca la importancia de los recursos sociales en el estrés aculturativo por encima de otras variables como el choque cultural o la nostalgia por el país de origen.

Palabras clave: persona sin hogar, exclusión social, Migración Internacional, impacto psicosocial, estrés aculturativo.

Abstract: In recent years has increased the number of homeless immigrants. The article shows the relationship between legal status and acculturative stress with sociodemographic variables,

Universidad Pontificia de Comillas 
social support, substance abuse, stressful life events and emotional distress in a sample of 107 homeless migrants. To this end has been made In-depth interviews and self-reports analyzing relationships between variables using various techniques of data analysis and content analysis. The results show the importance of the qualitative variable for «legal status» that has little correlation with the other variables. It also highlights the importance of social resources in acculturative stress over other variables such as culture shock or nostalgia for the country.

Keywords: homeless, social exclusion, Emigration and immigration, psychosocial Impact, acculturative stress.

\section{INTRODUCCIÓN}

\section{Algunos datos sobre el número de inmigrantes sin hogar}

España ha vivido lo que algunos autores han denominado como década prodigiosa de la inmigración (Aja, Arango, \& Oliver, 2009; Moreno \& Aierdi, 2011). Los datos hablan por sí solos, en España en 1998 el porcentaje de personas extranjeras era del 1,6\%, y doce años después, en 2010, era del 12,2\%. Se calcula un incremento de 5 millones de personas extranjeras en dicho período. Estos flujos han supuesto que España haya sido en el período del 2000 al 2008 el segundo país de la OCDE que más personas extranjeras ha recibido en términos absolutos, tan sólo por detrás de Estados Unidos, siendo el primero en términos relativos. En el actual momento de crisis económica los flujos se han ralentizado y algunos autores consideran que se está dando un cambio claro de tendencia.(Aja et al., 2009).

En paralelo al aumento de los flujos migratorios, se ha dado un incremento de las personas extranjeras en situación de exclusión social severa. Se trata de un fenómeno de escala europea que llevó a FEATNSA ${ }^{1}$ a declarar en el 2002 la situación

1 FEANTSA (Féderation Européene des Associations Nationales Travaillant Avec les Sans-Abri). Se trata de una organización que reúne a entidades de 29 países europeos que trabajan con personas sin hogar. Es un órgano consultivo del Consejo de Europa y de la Naciones Unidas. 
de las personas inmigrantes sin hogar como tema anual de investigación europeo (Edgar, Doherty, \& Meert, 2004). En el informe realizado para España (Cabrera \& Malgesini, 2002) se destacaba algunos datos como que el Centro Municipal de Acogida San Isidro (CASI), de la Comunidad de Madrid, atendió durante el año 2000 a un total de 2.031 personas distintas, de las cuales el 46,2\% eran extranjeras.

Los datos se mantienen a lo largo de los años como muestra la primera gran encuesta sobre población sin hogar realizada en España por el INE en el año 2005, con una estimación de 21.900 personas sin hogar para todo el territorio nacional, de las cuales un $48,2 \%$ eran extranjeras. La encuesta ha sido repetida en el año 2012 subiendo la cifra de personas sin hogar a 22.938 personas, de las cuales el 45,8\% son extranjeros, siendo el grupo mayoritario el de los africanos $(56,6 \%)$, seguido por los europeos $(22,3 \%)$ y los americanos $(15,2 \%)$.

Además de las encuestas del INE, en los últimos años las principales ciudades españolas han realizado recuentos nocturnos de personas sin hogar. La diferencia con las encuestas del INE es que éstas tan solo contabilizan a las personas que acuden a los recursos. En el recuento nocturno del País Vasco realizado de manera simultánea en las tres capitales (Fundación Eguía-Careaga, 2013) se localizaron 1.741 personas sin hogar (244 de ellas viviendo en la calle), siendo el 59,2\% extranjeras. En Barcelona la Xarxa d'Atenció a Personas Sense Llar (XAPSLL) realizó un recuento de las personas alojadas en sus equipamientos durante una noche concreta y, a su vez, el Servicio de Inserción Social (SIS) del Ayuntamiento de Barcelona con sus equipos de calle contó las personas que estaban durmiendo a la intemperie (Albert Sales i Campos, 2014). El SIS identificó 870 personas viviendo en la calle, con un 30\% de extranjeros provenientes de la Unión Europea y un 26,9\% de extranjeros no comunitarios. La XAPSLL identificó a 1.451 personas alojadas en sus recursos, de los cuales el 11,3\% eran personas con alguna nacionalidad de la UE y el 46;1\% eran no comunitarias. Por último, en la ciudad de Madrid, el foro técnico local para personas sin hogar de Madrid realizó un recuento nocturno a finales del 2013 (Foro técnico local para personas sin hogar de Madrid, 2013) localizando a 701 personas, con un $52 \%$ de personas extranjeras. 
TABLA 1

PROPORCIÓN DE EXTRANJEROS EN LA ENCUESTA DE INE Y RECUENTOS NOCTURNOS

\begin{tabular}{|l|c|c|c|c|}
\hline \multicolumn{1}{|c|}{$\%$} & INE (2012) & País Vasco (2013) & Barcelona (2013) & Madrid (2013) \\
\hline Españoles & 54,2 & 40,8 & 42,8 & 48 \\
\hline Extranjeros & $45,8 \%$ & 59,2 & 57,2 & 52 \\
\hline
\end{tabular}

A lo largo de los años se ha cambiado el foco de atención de las personas sin hogar, pasando de las características de las personas a la situación que están viviendo. Fruto de dicha evolución surge por parte de FEANTSA la necesidad de crear una tipología que delimitara qué es una persona sin hogar. Es el nacimiento de ETHOS (European Typology on Homelessness) cuya idea principal es distinguir dentro del sinhogarismo, entendido como un proceso de exclusión residencial más o menos severa, cuatro categorías principales o conceptuales ( $\sin$ techo, sin vivienda, vivienda insegura y vivienda inadecuada) que pueden subdividirse en trece categorías operativas, que a su vez pueden traducirse en diversas situaciones residenciales. Los datos presentados en los diversos recuentos nocturnos y encuestas del INE responden a las dos primeras categorías. La exclusión residencial vista en su dimensionalidad implicaría estudiar aspectos como las habitaciones de alquiler sin contrato, situaciones de hacinamiento, pensiones ilegales, vivir «de prestado» en casa de amigos y familiares, pisos que no reúnen condiciones de habitabilidad suficientes pese a ser alquilados, desahucios, etc. que han recibido la atención de algunos investigadores del Estado (Checa Olmos \& Arjona Garrido, 2006; Checa Olmos, 2007; Martínez Veiga, 1999) pero queda fuera de la presente investigación.

\section{La vulnerabilidad social de las personas migrantes}

¿Cuáles son los motivos de las cifras presentadas? ¿obedecen simplemente al aumento proporcional del número de personas extranjeras residentes en España o responden a una situación de mayor vulnerabilidad social del colectivo? Existen varios indicadores de referencia que nos muestran la vulnerabilidad social en la que se encuentran las personas migrantes. La tasa AROPE es 
el indicador europeo de riesgo de pobreza o exclusión social. Lo conforman tres situaciones: el riesgo de pobreza (60\% de la mediana de los ingresos por unidad de consumo); la carencia material severa (al menos cuatro conceptos de una lista de nueve); los hogares sin empleo o con baja intensidad en el empleo (hogares en los que sus miembros en edad de trabajar lo hicieron menos del 20\% del total de su potencial de trabajo durante el año de referencia). En el año 2013 la tasa de pobreza entre la población extranjera de fuera de la Unión Europea (en adelante UE) se situaba en el 47,8\% mientras que para los nacionales era del 16,5\%. (EAPN-España, 2014).

La encuesta de FOESSA (2014) nos permite ver la evolución entre el 2007 y el 2013 de la exclusión social en España, con un claro empeoramiento de la situación de las personas extranjeras. Los dos ámbitos donde se da una mayor exclusión son el empleo y la vivienda. En cuanto al primero, en el año 2013 el 59,3\% de los hogares con al menos un extranjero se habían visto afectados por la exclusión del empleo; 30,7 puntos más que en el año 2007 y 27,6 puntos por encima de los hogares españoles. En relación a la vivienda, el 48,5\% de los hogares con al menos un extranjero sufren exclusión de la vivienda, una cuarta parte más que los españoles cuyo porcentaje se sitúa en el $23,2 \%$. Tomando los datos globales se puede decir que el $24,4 \%$ de las personas extranjeras se encuentran en situación de exclusión severa por el 9,6\% de la población española o de la UE. Otro 28,3\% se encuentra en situación de exclusión moderada por el $12,8 \%$ de la población española o de la UE. Sumando ambos conceptos el 52,7\% de la población extranjera estás en situación de exclusión moderada o severa, llegando a cifras muy similares a la tasa AROPE.

Diversos autores afirman que el factor determinante para llevar a la exclusión extrema es la irregularidad administrativa que impide a las personas acceder al mercado de trabajo y a los recursos sociales en igualdad de condiciones que los nacionales. (Cabrera \& Malgesini, 2001; Sánchez \& Tezanos, 2004). Los inmigrantes están en desventaja respecto del resto de ciudadanos para poder salir de situaciones similares de desigualdad social y económica. Esta desventaja conforma el núcleo de la discriminación y debe tenerse en cuenta cuando se vincula inmigración y exclusión social. ¿cómo salir de condiciones de desigualdad si se parte de situaciones de desventaja? (Zapata-Barrero, 2004), es lo que lleva a algunos autores a situar el fenómeno de la inmigración bien como un eje transversal a los diversos factores de exclusión, o bien como un fenómeno 
estructural de la misma (Laparra, 2009; Raya, 2006.; Subirats et al., 2005), aunque tampoco debemos olvidar otros factores propios de la migración como la fragilidad de la red de apoyo o el estrés de iniciar una nueva vida en un país que no es el tuyo.

\section{METODOLOGÍA}

La investigación que se presenta analiza la existencia de factores psicosociales específicos que afectan a las personas migrantes sin hogar. Partimos de una de las variables más utilizadas en la psicología de la migración, el estrés aculturativo, y pretendemos medir su influencia en el tiempo que una persona duerme en la calle y su relación con las situaciones más extremas de la tipología ETHOS. También tomaremos la situación documental como una variable de exclusión. Las hipótesis de las que partimos son que el estrés aculturativo se relacionará con una mayor cronificación de la estancia en la calle y que la situación documental puede precipitar la exclusión residencia extrema.

El proceso seguido comienza con la búsqueda bibliográfica y el contacto con las asociaciones de apoyo a inmigrantes y organizaciones que trabajan con personas sin hogar. Previo a la investigación, existía un contacto fluido con ellas debido a que se trabaja en red en una plataforma a favor de las personas sin hogar, de la que el autor del artículo es colaborador habitual. En total se implicaron 16 entidades sociales.

La primera acción fue la creación de dos grupos de discusión con profesionales de los recursos y personas usuarias de los mismos para explorar algunas de las variables de investigación. A raíz de los resultados de estos grupos de discusión y de la bibliografía previa consultada se diseñó una entrevista semi-estructurada que fue testada entre las personas que participaron en el grupo de discusión de usuarios. Tras la versión final del cuestionario se procedió a la selección de la muestra siguiendo como criterio de estratificación el alojamiento actual:

- Personas que viven en la calle, estructuras temporales, edificios abandonados, etc. o bien combinando dichas situaciones con estancias cortas (menos de una semana) en recursos de alojamiento de emergencia.

- Personas que residen en alojamientos para persona sin hogar 
de media-larga estancia (tres o más meses de estancia) que han tenido historia reciente (menos de un año) de paso por las situaciones anteriormente descritas.

En total se pasaron 107 cuestionarios en los locales de las asociaciones, albergues, comedores sociales y centros de día de Bilbao. La muestra supone el 25\% del total de las personas migrantes sin hogar que había en la ciudad según el último recuento nocturno del año (Onartu, 2010). El cuestionario contenía las siguientes variables:

Variables sociodemográficas y de calle: sexo, edad, país de nacimiento, nivel de estudios, tiempo de estancia en España, y en Bilbao, motivos de la migración, situación documental, razón de no tener "papeles», empadronamiento, prestaciones sociales, tiempo trabajado, tiempo de estancia en la calle, tipos de alojamientos en los que ha estado.

Estrés aculturativo: se ha utilizado la escala Barcelona de estrés del inmigrante (BISS) (Tomás-Sabado, Qureshi, Antonin, \& Collazos, 2007) con 42 ítems y un formato de respuesta tipo Likert de 1 (totalmente en desacuerdo) a 4 (totalmente de acuerdo). La escala está compuesta por 4 factores: discriminación percibida, estrés o tensión del contacto intercultural, nostalgia y estrés psicosocial general.

Apoyo social: se ha utilizado una escala construida ad hoc basada en la versión adaptada a la población de inmigrantes africanos (Martínez, García, \& Maya, 1993) de la entrevista ASSIS (Barrera, 1980) para la parte funcional (satisfacción con el apoyo recibido), así como el cuestionario utilizado para medir el apoyo social en población sin hogar por Muñoz y Vázquez (Muñoz, Vázquez, \& Vázquez, 2003) para la parte estructural (frecuencia de contacto con diversas fuentes de apoyo).

Sucesos vitales estresantes: se ha construido un instrumento ad hoc basado en la adaptación y ampliación del LTE-Q (List of Threatening Experiences Questionnaire) (Brugha \& Cragg, 1990), realizado por Muñoz y Vázquez (Muñoz et al., 1995; Muñoz, Vázquez, \& Vázquez, 2003) y el inventario de experiencias extremas contenido en el cuestionario VIVO (Pérez-Sales et al., 2012).

Salud mental y abuso de sustancias: se ha utilizado el General Health Questionnaire (GHQ) (Goldberg \& Williams, 1988), la versión al castellano de Muñoz (Lobo, Muñoz, Goldberg, \& Williams, 2010). Se trata de la versión de 12 ítems (GHQ-12) con un formato de respuesta dicotómica (si/no). No mide específicamente psicopatología sino malestar psicológico y sufrimiento emocional. 
Para el abuso de sustancias se ha utilizado un cuestionario construido ad hoc basado en un sistema de indicadores de exclusión social (Vidal, Mota, Lázaro, Rubio, \& Iglesias, 2006). Explora la frecuencia y lugar de consumo del alcohol, así como con quién consume habitualmente. En cuanto al consumo de otras sustancias se pregunta por el tipo, la frecuencia y la vía de consumo.

Valoración del impacto vital: se ha realizado una adaptación a la realidad de las persona sin hogar de algunas escalas cortas del Cuestionario de Valoración de Impacto Vital Ontológico (VIVO) (Pérez-sales et al., 2012). Las escalas utilizadas son: futuro y esperanza, cambios en la identidad, cambio de prioridades, identidad de víctima, bondad del ser humano, confianza en el ser humano y aprendizaje.

Tras codificar las respuestas del cuestionario se procedió al análisis estadístico utilizando el programa SPSS 18.0, con un intervalo de confianza del $95 \%$ para todas las pruebas. Se han realizado pruebas bivariadas ( $\mathrm{T}$ de Student y ANOVA), así como correlaciones de Pearson para las variables continuas y Chicuadrado para las variables nominales. La confirmación de la validez de los instrumentos se ha realizado a través de análisis factorial exploratorio y confirmatorio (ecuaciones estructurales) y Alfa de Cronbach. Como forma de profundizar y matizar los resultados cuantitativos se hizo la transcripción de las partes donde se da una narrativa en las 107 entrevistas de los cuestionarios, complementándolo con 8 historias de vida. La selección de los casos ha respondido a su idoneidad para representar los distintos perfiles que se han visto a lo largo del proceso de investigación y su selección salió de las personas a las que se realizó el cuestionario. Para los análisis cualitativos se han seguido las tres etapas de la aplicación del análisis de contenido categorial siguiendo el método comparativo constante de Glaser (Elo \& Kyngäs, 2008; Glaser, 1965): codificación abierta, creación de categorías y abstracción.

\section{RESULTADOS}

En la exposición de los resultados se distinguirá entre las personas que en el momento de la entrevistas estaban viviendo en la calle, de las que lo hacían en un alojamiento de media-larga estancia, señalando aquellas diferencias que resulten significativas. 


\section{Descripción de la muestra}

La muestra total de personas que han sido entrevistadas son 107 con una mayoría de hombres $(97,2 \%)$. La media de edad es de 32 años, una población relativamente joven, siendo casi el $50 \%$ de la muestra menor de 30 años. Las personas que actualmente viven en un alojamiento para personas sin hogar son algo más jóvenes que las que viven en la calle, pero sin diferencias significativas. En cuanto a los lugares de procedencia destacan las personas procedentes del Magreb (el 44\% de la muestra total es de Marruecos), seguido de las de África Subsahariana.

Se observan algunas diferencias importantes en relación a la edad y la zona de procedencia. El 80\% de los jóvenes menores de 23 años que se encuentran en la calle proceden de la zona del Magreb, mayoritariamente de Marruecos (el 87,5\% de los menores de 23 años en calle procedentes de Marruecos), los jóvenes menores de 23 años procedentes de África Subsahariana se dividen entre Costa de Marfil, Ghana, Senegal y Somalia. La tendencia se repite entre los jóvenes con edades comprendidas entre los 24 y los 30 años, aunque en este caso se da un aumento importante de los procedentes de Argelia y se diversifican los países de procedencia de las personas de África Subsahariana. Las personas procedentes de Europa del Este, América Latina y Asia se concentran en los tramos de edad más altos.

El nivel de estudios de la mayoría son primarios, aunque hay una proporción significativa de personas que viven en un alojamiento para personas sin hogar con estudios secundarios. La media de tiempo residiendo en España es de 5 años y 7 meses, por lo que no estamos hablando de una población recién llegada, más del 60\% llevan más de 3 años residiendo en España. Casi la totalidad de las personas de la muestra están solteras o separadas.

El 86\% de las personas encuestadas planificaron poco o nada su venida a Bilbao, aunque eso no significa que no tuvieran un objetivo, un proyecto migratorio que va a influir en la percepción de la situación por la que están atravesando. Bien sea por la presión de la familia en origen al envío de dinero, por el sentido de la responsabilidad para con los seres queridos, o incluso por deudas contraídas en origen.

"Sostén ese personaje que manda dinero pagando de su bolsillo, bueno al menos estás en la calle pero estás haciendo lo que tienes que hacer, mantienes una dignidad. Hay gente que manda incluso todas 
las ayudas a su familia para poder mantener ese personaje, muchas veces se intenta salir de esa tensión mental con tóxicos,... es para volverse loco.»(España, 37 años; Grupo discusión profesionales)

TABLA 2

VARIABLES SOCIODEMOGRÁFICAS

\begin{tabular}{|c|c|c|c|}
\hline \multicolumn{2}{|r|}{$\%$} & Viven en calle & Recursos de alojamiento \\
\hline \multirow{5}{*}{ Edad } & 19-23 años & 16,7 & 21,3 \\
\hline & 24-30 años & 30 & 31,9 \\
\hline & 31-40 años & 31,7 & 27 \\
\hline & 41-50 años & 17,7 & 14,9 \\
\hline & Más de 50 años & 5 & 4,3 \\
\hline \multirow{5}{*}{$\begin{array}{l}\text { Lugar de } \\
\text { procedencia }\end{array}$} & Magreb & 65 & 63,8 \\
\hline & África Subsahariana & 21,7 & 34 \\
\hline & Latinoamérica & 8,3 & 2,1 \\
\hline & Europa del Este & 3,3 & 0 \\
\hline & Asia & 1,7 & 0 \\
\hline \multirow{6}{*}{ Nivel educativo } & Analfabeto & 0 & 2,1 \\
\hline & Leer y escribir & 6,7 & 4,3 \\
\hline & Profesionales & 20 & 14,9 \\
\hline & Primaria & 53,3 & 42,6 \\
\hline & Secundaria & 18,3 & 36,2 \\
\hline & Universidad & 1,7 & 0 \\
\hline \multirow{5}{*}{$\begin{array}{c}\text { Tiempo de } \\
\text { estancia en } \\
\text { España }\end{array}$} & Menos de 1 año & 6,7 & 10,6 \\
\hline & 1-3 años & 21,7 & 44,7 \\
\hline & 3-5 años & 25 & 19,1 \\
\hline & 5-7 años & 18,3 & 10,6 \\
\hline & Más de 7 años & 28,3 & 14,9 \\
\hline \multirow{4}{*}{ Estado civil } & Soltero & 83,3 & 87,2 \\
\hline & Casado & 5 & 4,3 \\
\hline & Viudo & 0 & 2,1 \\
\hline & Separado & 11,7 & 6,4 \\
\hline \multirow{5}{*}{$\begin{array}{l}\text { Planificación de } \\
\text { la migración }\end{array}$} & Nada & 73,3 & 59,6 \\
\hline & Poco & 13,3 & 25,5 \\
\hline & Algo & 5 & 12,8 \\
\hline & Bastante & 6,7 & 2,1 \\
\hline & Mucho & 1,7 & 0 \\
\hline \multirow{4}{*}{$\begin{array}{c}\text { Tiempo sin } \\
\text { hogar }\end{array}$} & Menos de 6 meses & 33,3 & 34 \\
\hline & Entre 6 meses y 1 año & 35 & 29,8 \\
\hline & 1-3 años & 21,7 & 25,5 \\
\hline & Más de 3 años & 10 & 10,6 \\
\hline
\end{tabular}


En lo referente al tiempo que se encuentran sin hogar el $66 \%$ lleva en la calle menos de un año, siendo el 33,7\% las que llevan más de un año. El tiempo medio es de 13 meses, aunque existe una alta variabilidad con una desviación típica de 18,9 meses. No se podría considerar una población cronificada en la situación pero tampoco unos recién llegados a la calle.

\section{Acceso a la ciudadanía y derechos sociales}

La cuestión de tener o no permiso de residencia y trabajo es una de las variables importantes en el proceso que puede llevar a una persona a dormir en la calle y también a salir de ella. La situación documental puede ser una vulnerabilidad más que dificulta el acceso a algunos recursos.

"Si esa persona sin hogar es de aquí, nacional, es más fácil todo que si no es de aquí, es más fácil porque te puedes poner en contacto con otras estructuras, porque ese señor tiene DNI y tiene un algo a lo que agarrarse y se puede hacer algo, pero si es de fuera todo es mucho más difícil.» (España, 43 años; Grupo discusión profesionales)

La mayoría de las personas entrevistadas son indocumentadas, aunque destacan el 21,6\% de las personas que tienen permiso de residencia y/o trabajo pero duermen en la calle. También es importante señalar las personas demandantes de asilo que están sin ningún apoyo social. A medida que aumenta el tiempo de residencia en España aumenta el porcentaje de personas que cuenta con algún tipo de permiso, de esta forma, el $50 \%$ de las personas que lleva entre 5 y 7 años residiendo en España cuenta con algún permiso.

La principal razón para encontrarse de manera irregular es la entrada sin autorización en el país (75\% de la muestra). Si ponemos este dato en relación con la media de tiempo residiendo en España (5 años y 7 meses), nos muestra las dificultades que tienen para cumplir los requisitos que les permita regularizar su situación por las vía del arraigo social. La segunda razón para encontrarse en situación administrativa irregular es haber perdido un permiso de trabajo y residencia previo. El análisis de contenido de los cuestionarios revela que se realizan importantes intentos de regularización y mantenimiento del permiso los que lo poseen. Las estrategias son 
buscar trabajo, cotizar uno mismo a la seguridad social aunque no disponga de empleo y recurrir a la compra de contratos invirtiendo todo el capital disponible.

"Yo cada mes cotizo a la seguridad social durante un año entero, cotizo 171 euros y por eso no puedo pedir las ayudas porque aparece como que estoy trabajando, pero en realidad pago esa cotización para poder renovar los papeles, pero resulta que en extranjería me han denegado la renovación. He hecho todo lo posible para no perder los papeles, incluso quedarme sin ayudas y al final los he perdido.»

(Marruecos, 42 años; Cuestionario 73)

TABLA 3

RESUMEN DIFERENTES MEDIDAS SITUACIÓN DOCUMENTAL

\begin{tabular}{|c|c|c|}
\hline \multicolumn{2}{|c|}{} & Situación documental \\
\hline \multirow{4}{*}{$\begin{array}{c}\text { Permiso administrativo } \\
(\%)\end{array}$} & Irregular & 72 \\
\cline { 2 - 3 } & Permiso de residencia & 5,6 \\
\cline { 2 - 3 } & Residencia y trabajo & 18,7 \\
\cline { 2 - 3 } & Nacionalidad española & 0,9 \\
\hline \multirow{3}{*}{$\begin{array}{c}\text { Motivos para } \\
\text { encontrarse irregular } \\
\text { (\%) }\end{array}$} & Solicitante de asilo & 2,8 \\
\cline { 2 - 3 } & Entrar sin autorización & 75,3 \\
\cline { 2 - 3 } & Entrar como turista & 2,6 \\
\cline { 2 - 3 } & Perder el permiso & 9,1 \\
\cline { 2 - 3 } & Entrar siendo menor de edad & 6,5 \\
\hline \multicolumn{2}{|c|}{ Otros } & 6,5 \\
\hline & Lugar donde vive ahora & 8,701 \\
\hline
\end{tabular}

${ }^{1}$ Chi-cuadrado

La posesión de un permiso de trabajo y residencia no es garantía de éxito en el proceso de inserción social. Las dificultades de renovación del permiso hace que, algunas personas, tengan trayectorias sociales descendentes como dos de los entrevistados en las historias de vida:

Sika es acogido por un centro de menores ya que hace la migración siendo muy joven (con apenas 14 años) con un recorrido de calle previo importante. Sin embargo, a pesar de contar con el permiso de residencia una serie de infortunios le llevan a perder la oportunidad de tener el permiso de trabajo y finalmente incluso perder el de residencia. 
"Al hacer el curso me mandaron de prácticas y ahí es donde me dieron una oportunidad buena. El jefe de quería contratar con 18 años (...), me dijo si tenía papeles, yo le dije que estaba esperando a que me contesten, pero me los denegaron, estuve con el abogado y eso pero nada. Yo creo que ahi es donde perdi mi oportunidad, ”

(Marruecos, 22 años; Historia de vida 8)

Los problemas de perder el permiso de residencia también se muestran en la historia de vida de Lahcen que tras una migración exitosa en la que consigue trabajar durante 4 años se queda en el paro, no consigue otro trabajo y no puede renovar los papeles.

"Me fui a Galicia y perdi el tiempo, el dinero y todo. Estuve dos años en Galicia, algunas veces trabajo y otras no, tenía permiso trabajo y residencia y al final lo perdí porque no puedo renovarlo por no tener trabajo ni contrato. Vivo cada día en un sitio, en casa de amigos, me dejan también un garaje grande que puedo dormir (...). Empiezo a beber mucho, todo el día, todos los días, de la mañana a la noche.»

(Sáhara, 44 años; Historia de vida 6)

A pesar de que el cuestionario no recogía entre sus preguntas la posesión o no de un pasaporte, el análisis de contenido de las entrevistas e historias de vida ha revelado que se trata de una variable importante a tener en cuenta más allá de la situación administrativa. La no posesión de un pasaporte deja a la persona en una posición de mayor vulnerabilidad que aquellas que se encuentran en situación irregular, impidiendo el acceso a cualquier tipo de prestación social más allá de recursos básicos de emergencia. Un ejemplo es André, un apátrida de facto que pese a llevar 23 años en España no tiene pasaporte al no reconocerle ni Angola ni Congo como ciudadano de su país. Dicha circunstancia ha marcado las escasas oportunidades de inserción social que ha tenido debido a los problemas de adicciones y con la justicia.

"Tenía una amiga que la conocí en Madrid, la chica tenía una casa, yo vivía con ella, vivíamos en Lavapiés, ella nació aquí y tenía los papeles y todo, no hay problema, y empecé a intentar conseguir el pasaporte en el consulado, pero no me querían dar el pasaporte, hasta hoy que estoy en Bilbao sin pasaporte y sigo durmiendo en la calle.»

(Angola, 45 años; Historia de vida 2)

Cuando se les pregunta a las personas las razones por las cuales se encuentran en la situación sin hogar la mayoría aduce la falta de 
dinero, trabajo y apoyo social vinculado a su situación de persona inmigrante sin papeles.

"Porque no tengo el apoyo de la gente porque no soy de aquí, ni estás con tu familia y te tienes que buscar la vida tu solo.»

(Marruecos, 22 años; Cuestionario 43)

«Si no tengo dinero, no tengo trabajo ¿Quévoy a hacer? Tampoco tengo papeles, ni familiares, no puedo hacer nada.»

(Senegal, 34 años; Cuestionario 68)

Los profesionales de la intervención también apuntan a estas mismas causas. Se señala que las personas migrantes suelen tener, a priori, menos problemáticas personales (abuso de sustancias y salud mental) que las autóctonas, por lo que la causa del sinhogarismo se asocia a factores externos como la falta de un permiso de trabajo y residencia o el propio proceso migratorio.

"La mayor parte de la gente de la que hablamos es una persona que en su país tenía más o menos familia, unas necesidades básicas algo cubiertas y que tenía lugar en su sociedad y acabar en la calle se debe al factor económico, es el determinante.»

(España, 39 años; Grupo discusión profesionales)

Sin embargo, aunque la cuestión de los papeles, el trabajo y la red de apoyo son fundamentales, las trayectorias hacia la exclusión extrema son variadas y en ocasiones condicionadas por otras variables como el consumo de alcohol y drogas en el país de origen. A Antonio un desgraciado accidente le lleva a la cárcel y ahí comienza una adicción a las drogas y el alcohol que desemboca en la calle durante casi una década. La no posesión del pasaporte le cierra todas las vías posibles para salir de la situación, y no es hasta el momento de la ayuda de una asociación cuando puede sacar el pasaporte y estabilizar un poco su situación.

"Aquí pedían en todo empadronamiento y no tenía ni el pasaporte porque estaba en Portugal, es más, tenía un nombre que ni siquiera era el mío porque tenía el miedo de que al dar mi nombre me llamaran para cumplir el resto de prisión, porque tenía la condicional y cada 30 días me tenía que presentar y yo no me había presentado (...). Me enganché otra vez a la droga, estuve en la calle desde el 2002 hasta 2008.»

(Cabo Verde, 42 años; Historia de vida 3) 
A pesar de los relatos, los datos no muestran diferencias significativas en el tiempo sin hogar entre las personas cuya situación documental se puede considerar precaria y estable. En ambos casos el tiempo medio de estancia en la calle es de 13,4 y 13,7 meses respectivamente. Tampoco se muestra relación entre la situación documental y otras variables como la frecuencia o satisfacción con las redes sociales (salvo con los profesionales de los recursos), el número de sucesos vitales estresantes padecidos, consumo de alcohol y drogas, malestar emocional y cambios en la identidad.

\section{Estrés aculturativo}

La escala de estrés aculturativo (escala BISS) puntúa de 0 a 3, siendo el cero totalmente en desacuerdo y el tres totalmente de acuerdo. Su puntuación media para la muestra es de 1,75, situándonos en puntuaciones de moderado acuerdo, lo cual implica un estrés aculturativo medio-alto. Sin embargo no todas las subescalas se comportan de la misma manera. La sub-escala de choque cultural tiene las puntuaciones más bajas $(1,2)$, la discriminación percibida y la nostalgia se sitúan en puntuaciones medias muy parecidas (1,8 y 1,9 respectivamente), y el estrés psicosocial general es la sub-escala que obtiene puntuaciones más altas $(2,3)$.

\section{TABLA 4}

CORRELACIONES DE LA ESCALA BISS CON LAS VARIABLES SOCIODEMOGRÁFICAS Y DE CALLE

\begin{tabular}{|c|c|c|c|c|c|}
\hline & $\begin{array}{l}\text { BISS } \\
\text { total }\end{array}$ & $\begin{array}{l}\text { Choque } \\
\text { cultural }\end{array}$ & $\begin{array}{c}\text { Discriminación } \\
\text { percibida }\end{array}$ & Nostalgia & $\begin{array}{c}\text { Estrés } \\
\text { psicosocial }\end{array}$ \\
\hline Media & 1,75 & 1,20 & 1,84 & 1,95 & 2,33 \\
\hline Desviación típica & 0,47 & 0,71 & 0,58 & 0,60 & 0,63 \\
\hline Edad $^{1}$ & $-0,115$ & $-0,131$ & $-0,225^{\star}$ & 0,084 & 0,017 \\
\hline Procedencia $^{2}$ & 0,307 & 2,355 & 0,513 & 0,149 & 0,306 \\
\hline Tiempo de estancia en España ${ }^{1}$ & $-0,037$ & $-0,037$ & $-0,081$ & 0,012 & 0,045 \\
\hline Documentación $^{2}$ & $2,255^{\star}$ & 2,142 & $2,406^{*}$ & 0,703 & 1,351 \\
\hline${\text { Empadronamiento }{ }^{2}}^{2}$ & 1,671 & 0,570 & 1,845 & 1,744 & $7,220^{\star \star}$ \\
\hline Tiempo padrón ${ }^{1}$ & $-0,259^{*}$ & $-0,162$ & $-0,226^{*}$ & $-0,128$ & $-0,333^{*}$ \\
\hline Prestaciones sociales $^{2}$ & 0,776 & 0,250 & 0,540 & 0,871 & $20,733^{\star \star}$ \\
\hline Tiempo en calle ${ }^{2}$ & 0,780 & 2,390 & 0,923 & 0,027 & 0,059 \\
\hline Tipo de alojamiento ${ }^{2}$ & $9,162^{*}$ & 1,888 & 3,249 & $4,100^{*}$ & $44,667^{\star *}$ \\
\hline
\end{tabular}

${ }^{*} p \leq 0,05 \quad{ }^{* *} p \leq 0,0015 \quad$ i: R de Pearson $\quad$ 2: ANOVA 
Las subescalas que tienen más influencia en las variables sociodemográficas son la discriminación percibida y el estrés psicosocial. En el caso de la primera, muestra relación con la media de edad, siendo las personas mayores las que sienten menos discriminación (posiblemente por tener estrategias de afrontamiento de la situación más eficaces). También guarda relación con el tiempo de empadronamiento con el que correlaciona negativamente, siendo reflejo no de un mayor tiempo de estancia en Bilbao (que no correlaciona con la discriminación percibida), sino de una mejor situación social que puede influir en una menor percepción de discriminación. A pesar de que también se ha mostrado una relación con el tipo de documentación, al comparar las medias de la escala global de estrés aculturativo y de la sub-escala de discriminación percibida agrupando la situación documental entre quienes tienen una situación documental precaria y estable, no se encuentran diferencias significativas siendo sus medias casi iguales.

La discriminación está bastante presente en las personas entrevistadas, el 50,5\% afirma que alguna vez ha sufrido agresiones o insultos racistas y en un $66,7 \%$ de los casos señalan que les ha afectado de alguna manera en su vida. No es posible delimitar si dichas situaciones de discriminación son debidas a su condición de persona extranjera o a la situación de pobreza extrema en la que se encuentran, aunque lo más probable es que se de una interacción entre ambas. Lo que queda en evidencia es que dichas situaciones pueden llegar a dificultar las posibilidades de ascenso social.

"Aquí hay mucha discriminación, hay personas que están cobrando la ayuda pero no pueden encontrar una casa sólo por su nacionalidad o por su piel y duermen en la calle.» (España, 37 años; Grupo discusión profesionales)

La subescala de estrés psicosocial se refiere a las condiciones del contexto que pueden provocar en una persona una demanda superior a su capacidad de respuesta. Las correlaciones muestran que las cuestiones que tienen que ver con el empadronamiento y acceso a ayudas sociales son especialmente significativas. 
TABLA 5

DIFERENCIA DE MEDIAS ESTRÉS PSICOSOCIAL CON BASE EN EMPADRONAMIENTO Y PRESTACIONES SOCIALES

\begin{tabular}{|l|l|c|c|}
\hline \multicolumn{2}{|c|}{} & $\begin{array}{c}\text { Media estrés } \\
\text { psicosocial }\end{array}$ & N \\
\hline \multirow{4}{*}{ Empadronamiento } & $\mathrm{Si}$ & 2,2 & 79 \\
\cline { 2 - 4 } & No & 2,6 & 28 \\
\hline \multirow{5}{*}{ Tipo padrón } & Pago por empadronamiento & 2,0 & 3 \\
\cline { 2 - 4 } & Favor de conocido o amigo & 2,8 & 10 \\
\cline { 2 - 4 } & Piso/habitación de familiar & 2,3 & 2 \\
\cline { 2 - 4 } & Piso/habitación alquilado & 1,3 & 7 \\
\cline { 2 - 4 } & Piso/habitación de asociación & 2,1 & 14 \\
\cline { 2 - 4 } & Padrón por servicios sociales & 2,3 & 43 \\
\hline \multirow{5}{*}{ Prestaciones sociales } & No recibe ayuda & 2,5 & 81 \\
\cline { 2 - 4 } & Ayuda Especial de Inserción & 2,1 & 10 \\
\cline { 2 - 4 } & RGl & 1,5 & 16 \\
\hline
\end{tabular}

Aquellas personas que no están empadronadas puntúan significativamente más alto que las que sí lo están en estrés psicosocial general. Además de las diferencias en la existencia de empadronamiento, también se han encontrado diferencias en el tipo de empadronamiento. El estrés psicosocial es significativamente menor $(F=5,428 \mathrm{p} \leq 0,001)$ entre quienes han conseguido el empadronamiento en un piso o habitación alquilado que los que lo están por el favor de un conocido o amigo o por servicios sociales, a pesar de que en este último caso dicho empadronamiento suponga un contacto con dichos servicios. También hay diferencias significativas entre estar empadronado en el piso de una asociación y por medio del favor de amigos y conocidos. Por último, el estrés psicosocial correlaciona con el tipo de prestaciones que se están recibiendo. Las diferencias son significativas $(\mathrm{F}=20,733 \mathrm{p} \leq 0,001)$ entre los que no reciben ninguna ayuda y los que reciben la Renta de Garantía de Ingresos (en adelante RGI), y entre estos últimos y los que reciben la Ayuda Especial de Inserción. Parece que a medida que aumenta la 
cantidad y estabilidad de las ayudas que se reciben disminuye el estrés psicosocial hasta parámetros intermedios.

TABLA 6

DIFERENCIAS DE MEDIAS EN LA ESCALA BISS CON BASE ENEL ALOJAMIENTO ACTUAL

\begin{tabular}{|l|c|c|}
\hline & Calle o recursos de emergencia & Alojamiento estable \\
\hline BISS total & 1,87 & 1,60 \\
\hline Sub-escala choque cultural & 1,28 & 1,10 \\
\hline Sub-escala discriminación percibida & 1,93 & 1,72 \\
\hline Sub-escala nostalgia & 2,05 & 1,81 \\
\hline Sub-escala estrés psicosocial & 2,64 & 1,94 \\
\hline
\end{tabular}

Las personas que en la actualidad siguen durmiendo en la calle o en recursos de emergencia alcanzan puntuaciones medias mayores tanto en el BISS total como en las sub-escalas. El estrés psicosocial general es especialmente relevante ya que las diferencias son significativas y la puntuación media de las personas que duermen en la calle está cercana a la puntuación máxima de 3. La nostalgia también ha resultado significativamente mayor entre las personas que duermen en la calle, resultando lógica una añoranza del país de origen viendo las condiciones sociales en las que se encuentran, en la mayoría de los casos peores que las del origen.

Si nos fijamos en las relaciones de la escala de estrés aculturativo con el resto de escalas obtenemos lo siguiente: 


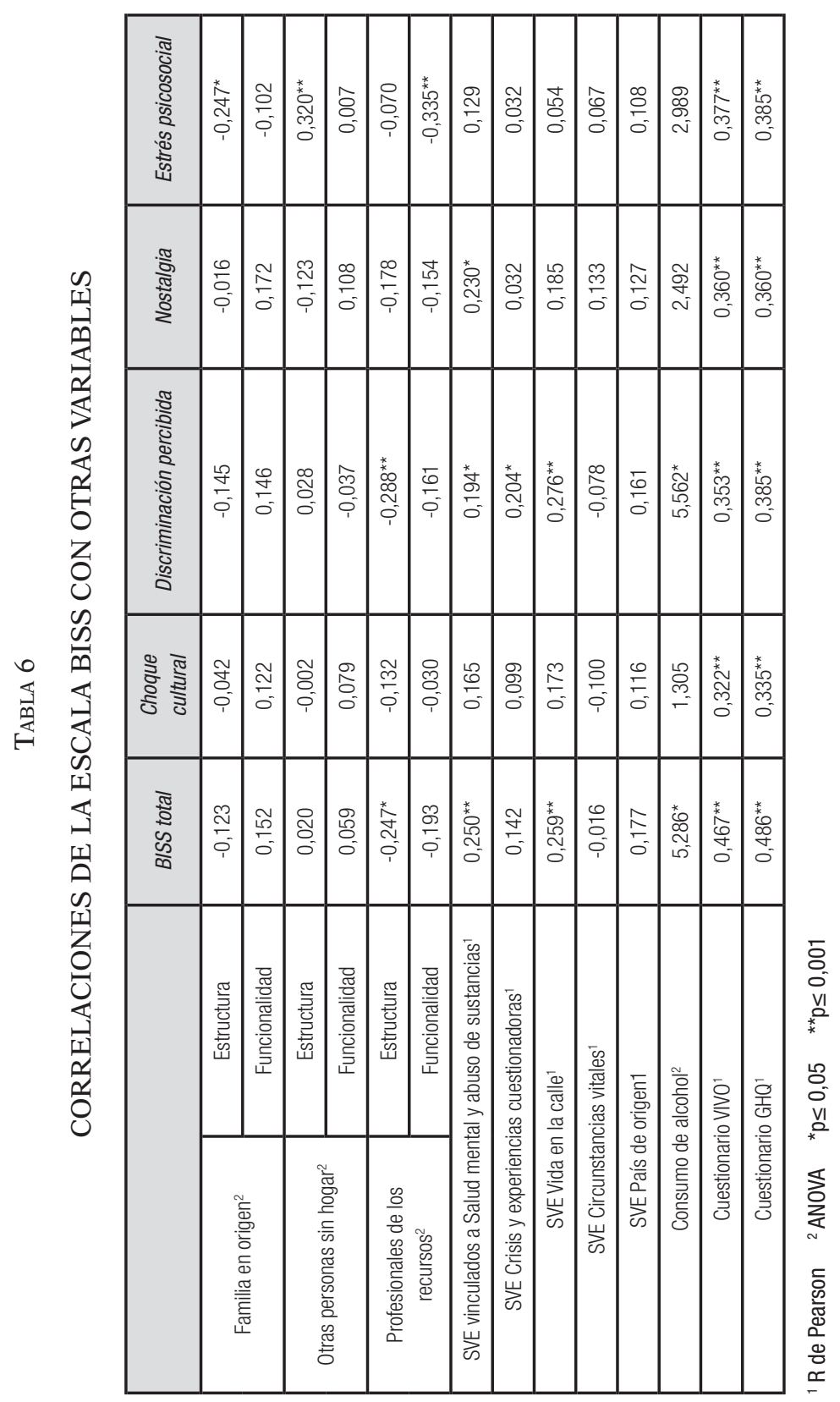


No parece que el apoyo social tenga una especial importancia en el estrés aculturativo. Destaca la frecuencia y satisfacción del contacto con los profesionales de los recursos. Las diferencias son significativas en la media global $(T=3,218 \mathrm{p} \leq 0,05)$ entre quienes tienen un contacto débil o nulo (media 2,17 ) y quienes tienen un contacto permanente (media 1,71), así como en la sub-escala de discriminación percibida $(\mathrm{T}=3,044 \mathrm{p} \leq 0,05)$ con diferencias todavía mayores (media 2,36 Vs media 1,78). En cuanto a la satisfacción con el apoyo, aquellas personas que están poco o nada satisfechos de su relación con los profesionales de los recursos puntúan significativamente más alto en estrés psicosocial $(\mathrm{T}=2,407 \mathrm{p} \leq 0,05)$ con una media de 2,60 , que quienes se encuentran satisfechos o muy satisfechos con la relación, con una media de 2,21. Resulta lógico al ser la fuente de apoyo que puede ofrecer mayores posibilidades de ayuda para afrontar la difícil situación que se vive.

«Para mí la gente que trabaja aquí es como si fuera mi familia.» (Marruecos, 57 años; Cuestionario 45)

Los datos muestran que la familia de origen actúa como modulador del estrés producido por la situación social, aunque las personas entrevistadas no consideran que su familia les pueda ayudar a salir de la situación en la que se encuentran.

«Ayuda material no, pero cuando hablo con ellos me quito cosas de la cabeza. Aunque no puedes decirle toda la verdad, a veces sí, por ejemplo sí le he dicho que duermo en la calle, un día se lo dije a mi hermana.»

(Sahara, 41 años; Cuestionario 31)

A pesar de la existencia de correlación, la prueba de comparación de medias no muestra una diferencia significativa entre quienes tienen un contacto débil o nulo (media 2,45 ) con la familia de origen y quienes tienen un contacto permanente (media 2,26). Lo contrario ocurre con las otras personas sin hogar $(\mathrm{T}=-2,976 \mathrm{p} \leq 0,05)$, cuyo mayor contacto implica más estrés psicosocial (media contacto permanente 2,42 Vs media contacto débil o nulo 2,00), debido quizás a la merma de relaciones sociales que se produce cuando una persona sólo se relaciona con otras en su misma situación, limitando las posibilidades de acceso a recursos. 
"Toda la gente que yo conozco viven en la calle, no conozco a nadie con casa.»

(Senegal 29 años; Cuestionario 15)

"Si no tienes andas con los que no tienen y si tienes andas con los que tienen. La gente que yo conozco está más o menos como yo.» (Marruecos, 23 años; Cuestionario 35)

Los resultados muestran la gran debilidad de las redes sociales de las personas migrantes sin hogar. Sus grandes apoyos son la familia, que no está presente, y los profesionales de los recursos de atención. Es difícil que ambos apoyos sean suficientes para afrontar las dificultades por las que pasan. Tal vez éste sea el motivo de la escasa representación entre las personas sin hogar de comunidades con redes sociales más amplias y sólidas como la latinoamericana o la china.

Los sucesos vitales estresantes no han mostrado correlaciones ni con el choque cultural ni con el estrés psicosocial general. Este último caso resulta más extraño porque era esperable una relación entre situación social y ocurrencia de sucesos vitales estresantes, tanto por el propio contexto de privación en el que el individuo se tiene que desenvolver, como por las menores posibilidades que dicho contexto brinda para acceder a recursos y estrategias de afrontamiento.

De aquellos que sí han correlacionado destacan los vinculados a la salud mental y el abuso de sustancias. A mayor ocurrencia de sucesos mayor discriminación percibida y mayor nostalgia del país de origen. $(F=2,492 p \leq 0,05)$. La misma lógica siguen los sucesos denominados como crisis y experiencias cuestionadoras (divorcio/ ruptura de relación afectiva estable, padecer problemas graves en el empleo, estar en prisión y haber estado en algún centro de menores) y con los sucesos vinculados a la vida en la calle (sufrir amenazas de muerte, agresiones o palizas, haber sido víctima de un asalto o robo y sufrir una agresión o insulto racista), en este caso mostrando diferencias significativas en la escala global $(\mathrm{F}=3,553 \mathrm{p} \leq 0,05)$ y la sub-escala de discriminación $(F=4,225 \mathrm{p} \leq 0,05)$, tal vez porque dichos sucesos guardan relación con actitudes y actos de población autóctona o, simplemente, porque generan una visión negativa de la sociedad en la que uno se encuentra. 
"Sí pasa, te llaman moro de mierda, sí te afecta, claro, pero ¿qué vas a hacer?»

(Argelia, 31 años; Cuestionario 30)

Encuantoalabuso de alcohol, la discriminación percibida sevuelve a revelar como una variable clave en la relación con las diferentes escalas utilizadas. En este caso, las personas abstemias perciben una menor discriminación (media 1,78 ) que aquellas que realizan un uso frecuente del alcohol (media 2,17), aunque las múltiples correlaciones existentes entre el uso de alcohol, discriminación percibida y el resto de escalas y variables sociodemográficas y de calle no permiten establecer una dirección causal unívoca. El estrés psicosocial pese a no resultar estadísticamente significativo, muestra tendencia a puntuaciones más altas según aumenta el consumo de alcohol.

Por último, la puntuación global del estrés aculturativo (BISS) correlaciona fuertemente con la escala del impacto vital (VIVO) y dentro de ésta, con la sub-escala de cambios en la identidad. Un mayor estrés generado por el cambio de país para las personas migrantes supone a su vez un mayor impacto identitario. La concepción del futuro y esperanza, así como la visión que se tiene del ser humano también se ven afectadas por el estrés aculturativo.

Los resultados nos muestran la dificultad de análisis de los factores diferenciales de las personas migrantes sin hogar. Si bien aspectos como la documentación, proyecto migratorio, acceso a recursos económicos y discriminación resultan más claros para entender por qué una personas migrante termina durmiendo en la calle, las múltiples interacciones que se producen en las variables analizadas complejizan la cuestión. Por poner un ejemplo, el cuestionario que hace referencia al malestar psicológico correlaciona con el estrés aculturativo y todas sus subescalas, pero eso no significa que el estrés aculturativo provoque dicho malestar, ya que puede ser provocado por la escasez de red social, o los sucesos vitales estresantes padecidos en el país de origen y aquí, el consumo de alcohol, que a su vez puede haber iniciado por la situación de dormir en la calle y no contar con una red social fuerte, etc. En cualquier caso vemos que al hablar de los factores psicosociales que influyen en el sinhogarismo de las personas migrantes es imprescindible introducir el estrés aculturativo entre ellos. 


\section{CONCLUSIONES}

Partimos de las hipótesis que nos habíamos planteado al comienzo de la investigación. Las personas migrantes sin hogar han obtenido puntuaciones moderadas en el estrés aculturativo, siendo las más altas las correspondientes al estrés psicosocial general (aquellos aspectos que tienen que ver con las condiciones objetivas de vida), y las más bajas las relacionadas con el choque cultural. Los datos muestran una relación entre el padecimiento de sucesos vitales estresantes vinculados a la salud mental y el abuso de sustancias, y el estrés aculturativo. A mayor número de sucesos padecidos mayor puntuación en la escala de estrés aculturativo. También se muestra una correlación positiva importante entre la puntuación en la escala de estrés aculturativo y la puntuación en la escala que mide malestar psicológico y sufrimiento emocional (escala GHQ) y la escala que mide el impacto vital de la experiencia de dormir en la calle (escala VIVO). Sin embargo, no se ha encontrado relación entre el estrés aculturativo y el consumo actual de alcohol. Tampoco se muestra relación entre el estrés aculturativo y la cronificación de la vida en la calle, hipótesis de la que partimos y que queda rechazada. Sí guarda relación en cambio con el alojamiento actual, fundamentalmente con la escala de estrés psicosocial, mostrando que las personas que actualmente siguen durmiendo en la calle tienen un mayor estrés psicosocial que las que duermen en un alojamiento estable.

$\mathrm{Si}$ analizamos el estrés aculturativo en el marco del modelo de estrés-proceso, considerando que la migración es estresante en la medida que es experimentada como tal por el individuo en relación con los recursos personales, sociales y materiales disponibles (Collazos et al., 2008), no nos ha de extrañar que el estrés aculturativo no guarde relación con una mayor cronificación de la vida en la calle y sí con el alojamiento actual, ya que éste último supone un cambio en los recursos sociales y materiales disponibles que influirá en una menor sensación de estrés. Los resultados concuerdan con la teoría del Síndrome de Ulises (Achotegui, 2006) que relaciona el estrés con las condiciones de vida en las que se tienen que desenvolver las personas y no tanto con las diferencias culturales. También apoyan la teoría de Dohrenwend (1978) que habla del estrés psicosocial como aquellos aspectos contextuales que rodean a la persona y adquieren vital importancia para explicar la reacción a los eventos estresantes. En este caso el estrés aculturativo actúa como parte del contexto 
social que influye en la disposición de recursos para enfrentar la situación y en la ocurrencia de eventos que demandan una mayor exigencia emocional, tal y como muestra la importante correlación de la escala BISS y las variables sociodemográficas vinculadas a la disponibilidad de recursos sociales y materiales.

De esta manera, los resultados nos muestran que el estrés aculturativo puede ser una variable a considerar en la exclusión social de la población migrante en tanto en cuanto se desvincule de las cuestiones más culturales y de adaptación a las normas sociales, usos y costumbres, y se vincule más al contexto político y social en el cual la persona se inserta. Dentro de esta visión cobra especial relevancia la actitud de la sociedad receptora para con las personas extranjeras. Los resultados de la investigación muestran importantes correlaciones entre la sub-escala de discriminación percibida y la ocurrencia de sucesos vitales estresantes, el consumo de alcohol, los cambios en la identidad y el malestar emocional. Las relaciones entre racismo, discriminación y exclusión social apenas han suscitado el interés académico en nuestro país salvo en momentos puntuales de violencia xenófoba (Martínez Veiga, 2001; Cachón, 2005) y a pesar de que, como hemos visto, la variable minoría étnica o lugar de procedencia está presente en todas las investigaciones sobre la exclusión social, el papel que juega en ello la discriminación y el racismo apenas ha sido investigado. Quizás el mayor reto es arrojar luz sobre si la discriminación conlleva procesos de exclusión social o simplemente responde a un intento de naturalizar situaciones de desventaja socioeconómica previas. En todo caso, es un hecho que vivimos en sociedades cada vez más diversas y desiguales y el futuro de la sociedad pasará, ineludiblemente, por cómo demos respuesta a ambos retos.

En cuanto al papel de la situación documental como precipitante de situaciones de exclusión, ha sido nombrada en el grupo de discusión de los profesionales de los recursos como uno de los factores externos de mayor vulnerabilidad que provoca que una persona extranjera termine en la calle e incluso dificulte la propia intervención social; las propias personas migrantes lo colocan como causa de su situación actual. Sin embargo, no se han encontrado correlaciones entre la situación documental y el tiempo de estancia en la calle. Tampoco con el alojamiento actual, el número de sucesos vitales estresantes, el consumo de alcohol y drogas, el malestar emocional o los cambios identitarios. A pesar de ello, la proporción 
de personas sin hogar en situación de irregularidad es muchísimo mayor que para la población general extranjera, y el análisis de contenido de las entrevistas y las historias de vida muestra que la inexistencia de un pasaporte coloca a la persona en una situación de vulnerabilidad total que le impide el acceso a aspectos básicos como el empadronamiento y las ayudas sociales, que sí han mostrado una importante correlación con variables tales como el tiempo de estancia en la calle o el malestar emocional. Quizá el pasaporte y la situación administrativa no lleva a las personas a la calle, pero sí condiciona las posibilidades de salir de ella, en algunos casos determina la relación que se establece con la familia de origen al no poder cumplir las expectativas generadas y convierte las equivocaciones que puede sufrir cualquier persona en su proceso vital (como extraviar el pasaporte o no renovar la documentación) en errores fatales con consecuencias en los procesos de inserción.

El contexto exclusógeno que opera en las personas migrantes pasa por los cambios en el sistema económico y social que obliga a migrar a millones de personas, facilitando a su vez la entrada de mano de obra barata en los países receptores. Tampoco se puede obviar la existencia de una ley que al limitar el acceso a derechos se convierte en una ley exclusógena, la Ley de extranjería, que determina aquellos derechos de los que gozan las personas que cuentan con un permiso de residencia y/o trabajo en el país, quedando por lo tanto las personas que no poseen dichos permisos fuera de ellos. Esa diferenciación marca el acceso a la ciudadanía o, cuanto menos, la segmentación en unos ciudadanos que podríamos denominar «de primera» y «de segunda». La crítica no es baladí, ya que los diversos estudios sobre los factores de exclusión social en España (Laparra, 2009; Raya, 2007), sitúan el fenómeno de la inmigración como un eje trasversal a los diversos factores de exclusión, o como un fenómeno estructural de la misma.

En todos los casos la situación administrativa se muestra como un escalón imprescindible en el ascenso social, desde los primeros pasos para conseguir el pasaporte pasando por el empadronamiento (para el cual es imprescindible el padrón), las ayudas sociales (para las cuales es necesario el empadronamiento y en algunos casos el permiso de residencia), el permiso de residencia y por último el de trabajo. Aunque dicha la escalera sirve como metáfora para representar el proceso a seguir no se trata de un ascenso gradual. Las propias historias de vida muestra idas y venidas, momentos 
en los que se consigue el permiso de residencia y trabajo y luego se pierde, ayudas que son quitadas, etc. Entra en juego muchos factores (como el abuso de alcohol y drogas tan presente en algunas de las historias mostradas) y otros que deben ser analizados en profundidad, aunque no cabe duda que el pasaporte y el permiso de residencia y trabajo juegan un papel fundamental en los procesos de exclusión social de las personas inmigrantes.

Podríamos considerar la situación documental como una variable que acompaña al bienestar de las personas (Blanco \& Díaz, 2006) que provoca un acceso diferenciado a los recursos disponibles y termina incidiendo en un mayor impacto de los eventos. Más allá del permiso de residencia y trabajo, las cuestiones burocráticas que las personas migrantes deben superar en el país y que en los autóctonos apenas tiene incidencia, adquieren especial relevancia en su vida. Un ejemplo puede ser el empadronamiento que juega un papel fundamental, a través de la accesibilidad a las ayudas sociales, en la posibilidad de conseguir un alojamiento estable. En la investigación las prestaciones sociales muestran diferencias significativas en el tipo de alojamiento actual, el tiempo en calle, la frecuencia de la relación con otras personas sin hogar y el estrés psicosocial general. Ninguna persona que recibe prestaciones sociales sigue durmiendo en la calle, lo cual no quiere decir que la mera concesión de una ayuda económica vacíe las calles de personas sin hogar, pero es cierto que sin las prestaciones sociales es muy difícil salir de la calle.

Las dificultades mostradas en el presente estudio para «visibilizar estadísticamente» la influencia de la situación documental a pesar de la relevancia que adquiere en las narrativas de las personas y algunas de las historias de vida nos refuerza en la importancia de tener en cuenta los factores estructurales en el estudio de la exclusión social, también desde la psicología, y nos debe de poner alerta sobre la manera de investigar el mundo social, valorando las aportaciones que la perspectiva cualitativa nos puede ofrecer. Quedan abiertas líneas de investigación con amplio recorrido como la influencia de las redes sociales en el sinhogarismo o las estrategias de afrontamiento que utilizan las personas para salir de la situación en la que se encuentran. Esperemos que estos temas no queden fuera de la agenda de la academia y podamos aportar soluciones que mejoren la calidad de la vida de la gente. 


\section{BIBLIOGRAFÍA}

Achotegui, J. (2006): «Estrés límite y salud mental: el síndrome del inmigrante con estrés crónico y múltiple (síndrome de Ulises)». Migraciones, (19), pp. 59-85.

Aja, E., Arango, J., \& Oliver, J. (2009): La inmigración en tiempos de crisis. Anuario de la inmigración en España. Barcelona, Fundació CIDOB.

BARRERA, M. (1980): "A method for the assessment of social support networks in community survey research». Connections, (3), pp. 8-13.

Blanco, A. \& DíAz, D. (2006): «Orden social y salud mental: Una aproximación desde el bienestar social». Clínica y Salud, 17(1), pp. 7-29.

Brugha, T. S. \& CRAGG, D. (1990). «The List of Threatening Experiences: the reliability and validity of a brief life events questionnaire». Acta Psychiatrica Scandinavica, 82(1), pp. 77-81.

Cabrera, P. \& Malgesini, G. (2002): Inmigrantes y sinhogarismo en España. Informe Nacional.

Cabrera, P., Rubio, M. J. \& Blasco, J. (2008): ¿Quién Duerme en la Calle? Una investigación social y ciudadana sobre las personas sin techo. Una Investigación Social y Ciudadana Sobre las Personas sin Techo. Barcelona, Fundació Caixa Catalunya.

Cachon, L. (2005): Bases sociales de los sucesos de Elche de septiembre de 2004: crisis industrial, inmigración y xenofobia. Ministerio de Trabajo e inmigración.

Checa Olmos, J. \& Arjona Garrido, A. (2006): «Inmigración y segregación residencial. Aproximación teórica y empírica para el caso almeriense». Migraciones, (20), pp. 143-171.

Checa Olmos, J. C. (2007): Viviendo juntos aparte: la segregación espacial de los Africanos en Almería. Barcelona, Icaria.

Collazos, F., Qureshi, A., Antonín, M. \& Tomás-Sábado, J. (2008): «Estrés aculturativo y salud mental en la población inmigrante». Papeles del Psicologo, 29(3), pp. 307-315.

EAPN-ESPAÑA (2014). Seguimiento del indicador de riesgo de pobreza y exclusión social en España 2009-2013, Madrid, Red Europea de Lucha contra la Pobreza y la Exclusión Social

Edgar, B., Doherty, J. \& Meert, H. (2004): Immigration and Homelessness in Europe - FEANTSA Research. FEANTSA Foundation.

Elo, S. \& KYngäs, H. (2008): «The qualitative content analysis process». Journal of advanced nursing, 62(1), 107-15.

FEANTSA (2002): Immigration and homelessness in the European Union. Analysis and overview of the impact of immigration on homeless services in the European Union.

Foro técnico local PARA PERSONAS SIN HOGAR DE MADRID (2013): Operación de recuento nocturno de personas viviendo sin techo en las calles de Madrid. Principales resultados. Ayuntamiento de Madrid. 
FUNDACIÓN FOESSA. (2014): VII Informe sobre exclusión y desarrollo social en España. Madrid, Fundación Foessa.

Fundación Eguía-CAREAga (2013): Cuantificación y caracterización de la exclusión residencial en las capitales de la CAPV. Donosti, SIIS Centro de Documentación y Estudios.

Glaser, B. G. (1965): «The Constant Comparative Method of Qualitative Analysis». Social Problems, 12(4), pp. 436-445.

Goldberg, D. P. \& Williams, P. (1988): A user's guide to the General Health Questionnaire. Windsor, Berks, NFER-Nelson.

INE. (2012): Encuesta sobre las personas sin hogar (personas). Año 2012. Madrid, Instituto Nacional de Estadística.

KrIPPEndorfF, K. (2013): Content Analysis: An Introduction to Its Methodology (p. Philadelphia). SAGE.

LAPARRA, M. (2009): La exclusión social en España: un espacio diverso y disperso en intensa transformación. VI Informe sobre exclusión y desarrollo social en España 2008. Madrid, Fundación FOESSA.

Lobo, A., Muñoz, P., Goldberg, D. \& Williams, P. (2010): Cuestionario de salud general GHQ (General Health Questionnaire). Versiones en lengua española validadas. GHQ (General Health Questionnaire). Barcelona, Masson.

Martínez García, M. F., García Ramírez, M. \& Maya Jariego, I. (2001): «El efecto amortiguador del apoyo social sobre la depresión en un colectivo de inmigrantes». Psicothema, 13(4), pp. 605-610.

Martínez, M., García, M. \& Maya, I. (1993): Pretest del cuestionario de evaluación de necesidades y recursos, y del ASSIS de Barrera. Sevilla.

Martínez Veiga, U. (1999): Pobreza, segregación y exclusión espacial: la vivienda de los inmigrantes extranjeros en España. Barcelona, Icaria.

Martínez Veiga, U. (2001): El Ejido: discriminación, exclusión social y racismo, Madrid, Libros de la Catarata.

Moreno, G. \& Aierdi, X. (2011): Anuario de la inmigración en el País Vasco 2010. Bilbao, Ikuespegiak - Observatorio Vasco de Inmigración.

Muñoz, M., Vázouez, C. \& CRuzado, J. A. (1995): Personas sin hogar en Madrid: Informe psicosocial y epidemiológico. Madrid, Comunidad de Madrid.

Muñoz, M., Vázouez, C. \& VÁzouez, J. J. (2003): Los límites de la exclusión: Estudio sobre los factores económicos psicosociales y de salud que afectan a las personas sin hogar en Madrid. Madrid, Ediciones Témpora.

ONARTU. (2010): Análisis de situación de las personas en situación de exclusión residencial grave. Bilbao.

Pérez-Sales, P., Eiroa-Orosa, F. J., Olivos, P., Barbero-Val, E., FernándezLiria, A. \& Vergara, M. (2012): "Vivo Questionnaire: A Measure of Human Worldviews and Identity in Trauma, Crisis, and LossValidation and Preliminary Findings». Journal of Loss and Trauma, 17(3), pp. 236-259.

RAYA, E. (2007): «Exclusión social: Indicadores para su estudio y aplicación 
para el trabajo social». Revista del Ministerio deTrabajo y Asuntos sociales, 70, pp. 155-172.

Sales i Campos, A. (2014): Diagnosis 2013. Las personas sin hogar en la ciudad de Barcelona y la evolución de los recursos de la Red de Atención a Personas sin Hogar. Barcelona, Xarxa d'Atenció a Personas Sense Llar.

Sánchez, M. R. \& Tezanos, S. (2004): «Los inmigrantes «sin hogar» en España: un caso extremo de exclusión social». Revista del Ministerio de Trabajo y Asuntos Sociales, 55, pp. 45-64.

SIIS Centro de Documentación y Estudios. (2013): Estudio sobre la situación de las personas en situación de exlusión residencial grave en la CAPV. Vitoria - Gasteiz, Eusko Jaurlaritza-Gobierno Vasco.

Subirats, J. (2004). Pobreza y exclusión social. Un análisis de la realidad española y europea. (Colección Estudios Sociales no 16, Ed.). Barcelona, Fundación La Caixa.

Subirats, J., Gomà, R. \& Brugué, Q. (2005): Análisis de los factores de exclusión social. Documentos de trabajo (Fundación BBVA). Barcelona, Fundación BBVA.

Tomás-Sabado, J., Qureshi, A., Antonin, M. \& Collazos, F. (2007): «Construction and preliminary validation of the Barcelona Immigration Stress Scale». Psychological Reports, 100(3 Pt 1), pp. 1013-1023.

Vidal, F., Mota, R., Lázaro, S., Rubio, E., \& Iglesias, J. (2006): Ensayo de sistema de indicadores sobre exclusión social. La exclusión social y el estado de bienestar en España. Barcelona, Icaria.

Zapata-BARrero, R. (2004): Multiculturalidad e inmigración. Madrid, Síntesis. 
